\title{
Research Article \\ Bending Analysis of Functionally Graded Plates in the Context of Different Theories of Thermoelasticity
}

\author{
A. M. Zenkour, ${ }^{1,2}$ D. S. Mashat, ${ }^{1}$ and K. A. Elsibai ${ }^{3}$ \\ ${ }^{1}$ Department of Mathematics, Faculty of Science, King AbdulAziz University, P.O. Box 80203, \\ Jeddah 21589, Saudi Arabia \\ ${ }^{2}$ Department of Mathematics, Faculty of Science, Kafrelsheikh University, Kafr El-Sheikh 33516, Egypt \\ ${ }^{3}$ Department of Mathematics, Faculty of Applied Science, Umm Al-Qura University, P.O. Box 715, \\ Holy Makkah, Saudi Arabia
}

Correspondence should be addressed to A. M. Zenkour, zenkour@gmail.com

Received 7 April 2009; Revised 15 October 2009; Accepted 25 November 2009

Recommended by Mehrdad Massoudi

The quasistatic bending response is presented for a simply supported functionally graded rectangular plate subjected to a through-the-thickness temperature field under the effect of various theories of generalized thermoelasticity, namely, classical dynamical coupled theory, Lord and Shulman's theory with one relaxation time, and Green and Lindsay's theory with two relaxation times. The generalized shear deformation theory obtained by the first author is used. Material properties of the plate are assumed to be graded in the thickness direction according to a simple exponential law distribution in terms of the volume fractions of the constituents. The numerical illustrations concern quasistatic bending response of functionally graded square plates with two constituent materials are studied using the different theories of generalized thermoelasticity

Copyright (C) 2009 A. M. Zenkour et al. This is an open access article distributed under the Creative Commons Attribution License, which permits unrestricted use, distribution, and reproduction in any medium, provided the original work is properly cited.

\section{Introduction}

In the recent years, functionally graded materials (FGMs) have gained considerable attention in many engineering applications. FGMs are considered as a potential structural material for future high-speed spacecraft and power generation industries. FGMs are new materials, microscopically inhomogeneous, in which the mechanical properties vary smoothly and continuously from one surface to the other [1-7].

The effect of thermal loading on the displacement and stress fields for FGM plates and shells has been studied by a number of authors. For example, Wetherhold et al. [5] have considered the use of functionally graded materials to eliminate or control thermal 
deformation in beams and plates. Suresh and Mortensen [6] have discussed the large deformation of graded multilayered composites under mechanical and thermal loads. Praveen and Reddy [7] have investigated the response of functionally graded ceramic-metal plates using a finite element that accounts for the transverse shear strains, rotary inertia, and moderately large rotations in the von Karman sense.

The theory of thermoelasticity that includes the effect of temperature change has been well established. According to this theory, the temperature field is coupled with the elastic strain field. This theory covers a wide range of extensions of classical dynamical coupled thermoelasticity. Lord and Shulman [8] and Green and Lindsay [9] have extended the coupled theory of thermoelasticity by introducing the thermal relaxation times in the constitutive equations. Additional thermoelasticity theories have been presented and investigated by other researchers [10-22].

Lord and Shulman [8] have considered isotropic solids and introduced one relaxation time parameter into the Fourier heat conduction equation; that is, both the heat flux and its time derivative are considered in the heat conduction equation. The heat equation associated with this theory is thus hyperbolic. A direct consequence is that the paradox of infinite speed of propagation inherent in both the uncoupled and coupled theories of classical thermoelasticity is eliminated and the heat wave feature can be modeled by the generalized thermoelasticity. The Green and Lindsay's theory [9] does not violate the Fourier's law of heat conduction when the body under consideration has a center of symmetry. In this theory, both the equations of motion and heat conduction are hyperbolic but the equation of motion is modified and differs from that of classical coupled thermoelasticity theory.

In this paper, a generalized nonclassical dynamic coupled thermoelasticity analysis is carried out on a functionally graded material plate. Through-the-thickness temperature distribution varying according to exponential law is considered and then temperature and stress behavior are presented for the mentioned plate. The governing equations of FGM plate for two-dimensional generalized thermoelastic problems are derived within the framework of the classical coupled theory, Lord and Shulman's theory, and Green and Lindsay's theory. The material properties of the functionally graded plate are assumed to vary continuously through the thickness according to an exponential law distribution of the volume fraction of the constituents. A generalized shear deformation theory is presented to obtain the governing equations. An exact solution for the coupled governing equations under simply supported boundary conditions is obtained. Numerical results are provided to show the influence of the material properties, and a temperature field on the displacement and stresses.

\section{Formulation of the Problem}

We consider a solid rectangular plate of length $a$, width $b$, and thickness $h$, made of functionally graded materials. The material properties of the FGM plate are assumed to be function of the volume fraction of the constituent materials. Using the rectangular Cartesian coordinates $(x, y, z)$ we take the functional graded between the physical properties and $z$ for ceramic and metal FGM plate

$$
P(z)=P_{m} e^{\eta_{P}((2 z+h) / 2 h)}, \quad \eta_{p}=\ln \left(\frac{P_{c}}{P_{m}}\right)
$$

where $P_{c}$ and $P_{m}$ are the corresponding properties of ceramic (top surface) and metal (bottom surface), respectively. 
The displacements of a material point located at $(x, y, z)$ in the plate may be written as

$$
\begin{gathered}
u_{x}(x, y, z, t)=u-z \partial_{x} w+\Psi \varphi_{x} \\
u_{y}(x, y, z, t)=v-z \partial_{y} w+\Psi \varphi_{y} \\
u_{z}(x, y, z, t)=w
\end{gathered}
$$

where $\Psi=\hbar \sin (\pi z / h), \hbar=h / \pi$, and $\partial_{x}, \partial_{y}$ represent the differentiation with respect to $x$ and $y .\left(u_{x}, u_{y}, u_{z}\right)$ are the displacements corresponding to the co-ordinate system and are functions of the spatial co-ordinates, $(u, v, w)$ are the displacements along the axes $x, y$, and $z$, respectively, and $\varphi_{x}$ and $\varphi_{y}$ are the rotations about the $y$ - and $x$-axes.

The strain components will be

$$
\begin{gathered}
\varepsilon_{x}=\varepsilon_{x}^{0}+z \varepsilon_{x}^{1}+\Psi \varepsilon_{x}^{2}, \\
\varepsilon_{y}=\varepsilon_{y}^{0}+z \varepsilon_{y}^{1}+\Psi \varepsilon_{y^{\prime}}^{2} \\
\varepsilon_{x y}=\varepsilon_{x y}^{0}+z \varepsilon_{x y}^{1}+\Psi \varepsilon_{x y^{\prime}}^{2} \\
\varepsilon_{z}=0, \quad \varepsilon_{y z}=\Psi^{\prime} \varphi_{y}, \quad \varepsilon_{x z}=\Psi^{\prime} \varphi_{x},
\end{gathered}
$$

where

$$
\begin{array}{cl}
\varepsilon_{x}^{0}=\partial_{x} u, \quad \varepsilon_{y}^{0}=\partial_{y} v, & \varepsilon_{x y}^{0}=\partial_{x} v+\partial_{y} u, \\
\varepsilon_{x}^{1}=-\partial_{x}^{2} w, \quad \varepsilon_{y}^{1}=-\partial_{y}^{2} w, & \varepsilon_{x y}^{1}=-2 \partial_{x} \partial_{y} w, \\
\varepsilon_{x}^{2}=\partial_{x} \varphi_{x}, \quad \varepsilon_{y}^{2}=\partial_{y} \varphi_{y}, \quad \varepsilon_{x y}^{2}=\partial_{x} \varphi_{y}+\partial_{y} \varphi_{x} .
\end{array}
$$

\section{Theories of Thermoelasticity}

In addition, the stress-strain-temperature relations for the linear thermoelastic materials are given, according to the generalized theories of thermoelasticity, by

$$
\sigma_{i j}=c_{i j k l} \varepsilon_{k l}-\beta_{i j}\left(T+t_{1} \dot{T}-T_{0}\right)
$$

where $T, T_{0}, \varepsilon_{i j}, \beta_{i j}, c_{i j k l}$, and $t_{1}$ are the absolute temperature, reference temperature, strain tensor components, components of tensor of stress-temperature moduli, components of tensor of elastic moduli, and the first relaxation time of Green and Lindsay's theory, respectively. 
In details, we can rewrite the stress components in the deferent theories of generalized thermoelasticity as follows:

$$
\begin{gathered}
\sigma_{x}=E^{1} \varepsilon_{x}+E^{2} \varepsilon_{y}-\beta\left(T+t_{1} \dot{T}-T_{0}\right), \\
\sigma_{y}=E^{1} \varepsilon_{y}+E^{2} \varepsilon_{x}-\beta\left(T+t_{1} \dot{T}-T_{0}\right), \\
\sigma_{z}=0, \quad \sigma_{y z}=E^{3} \varepsilon_{y z}, \quad \sigma_{x z}=E^{3} \varepsilon_{x z}, \quad \sigma_{x y}=E^{3} \varepsilon_{x y},
\end{gathered}
$$

where

$$
E^{1}=\frac{E}{1-v^{2}}, \quad E^{2}=\frac{E v}{1-v^{2}}, \quad E^{3}=\frac{E}{2(1+v)}
$$

and the material properties $E$ and $v$ are functions of $z$. In the absence of body forces and internal heat generation, the heat conduction equation will be in the form

$$
\left(\kappa T_{, j}\right)_{, j}-\rho c\left(\dot{T}+t_{2} \ddot{T}\right)-\beta T_{0}\left(\dot{u}_{j}+t_{3} \ddot{u}_{j}\right)_{, j}=0,
$$

where $t_{2}$ and $t_{3}$ are additional relaxation times. A comma followed by index $j$ denotes partial differentiation with respect to the position $x_{j}$ of a material particle. A superimposed dot indicates partial derivative with respect to time $t$. In addition to the elastic coefficients $E$, and $v$, the material properties $\kappa, \rho, c$, and $\beta$ are also functions of $z$.

It is clear that, by setting $t_{1}=0$ in (3.2) and $t_{2}=t_{3}=0$ in (3.4), we get the field equations for the conventional coupled theory of thermoelasticity; whereas when $t_{1}=0$ and $t_{2}=t_{3} \neq 0$, the equations reduce to the Lord and Shulman's theory and when $t_{3}=0$ and $t_{1}$ and $t_{2}$ are nonvanishing, the equations reduce to the Green and Lindsay's theory.

Note that the stress-temperature modulus $\beta$ is given in terms of Young's modulus $E$, Poisson's ratio $v$, and the thermal expansion coefficient $\alpha$ by the relation

$$
\beta=\frac{\alpha E}{1-2 v}
$$

Generally, this study assumes that Young's modulus E, Poisson's ratio $v$, material density $\rho$, thermal expansion coefficient $\alpha$, specific heat capacity $c$, and thermal conductivity coefficient $\kappa$ of the FGM change continuously through the thickness direction of the plate and obey the gradation relation given in (2.1).

\section{Solution of the Problem}

To solve the problem, we obtain the stress and moment resultants for the FGM plate by integrating the stress components given in (3.2) over the thickness and written as 


$$
\begin{aligned}
& N_{x}=A_{1}^{1} \varepsilon_{x}^{0}+A_{1}^{2} \varepsilon_{y}^{0}+A_{2}^{1} \varepsilon_{x}^{1}+A_{2}^{2} \varepsilon_{y}^{1}+A_{3}^{1} \varepsilon_{x}^{2}+A_{3}^{2} \varepsilon_{y}^{2}-B_{1}\left(T+t_{1} \dot{T}-T_{o}\right), \\
& N_{y}=A_{1}^{1} \varepsilon_{y}^{0}+A_{1}^{2} \varepsilon_{x}^{0}+A_{2}^{1} \varepsilon_{y}^{1}+A_{2}^{2} \varepsilon_{x}^{1}+A_{3}^{1} \varepsilon_{y}^{2}+A_{3}^{2} \varepsilon_{x}^{2}-B_{1}\left(T+t_{1} \dot{T}-T_{o}\right), \\
& N_{x y}=A_{1}^{3} \varepsilon_{x y}^{0}+A_{2}^{3} \varepsilon_{x y}^{1}+A_{3}^{3} \varepsilon_{x y^{\prime}}^{2} \\
& M_{x}=A_{2}^{1} \varepsilon_{x}^{0}+A_{2}^{2} \varepsilon_{y}^{0}+A_{4}^{1} \varepsilon_{x}^{1}+A_{4}^{2} \varepsilon_{y}^{1}+A_{5}^{1} \varepsilon_{x}^{2}+A_{5}^{2} \varepsilon_{y}^{2}-B_{2}\left(T+t_{1} \dot{T}-T_{o}\right), \\
& M_{y}=A_{2}^{1} \varepsilon_{y}^{0}+A_{2}^{2} \varepsilon_{x}^{0}+A_{4}^{1} \varepsilon_{y}^{1}+A_{4}^{2} \varepsilon_{x}^{1}+A_{5}^{1} \varepsilon_{y}^{2}+A_{5}^{2} \varepsilon_{x}^{2}-B_{2}\left(T+t_{1} \dot{T}-T_{o}\right), \\
& M_{x y}=A_{2}^{3} \varepsilon_{x y}^{0}+A_{4}^{3} \varepsilon_{x y}^{1}+A_{5}^{3} \varepsilon_{x y}^{2} \\
& S_{x}=A_{3}^{1} \varepsilon_{x}^{0}+A_{3}^{2} \varepsilon_{y}^{0}+A_{5}^{1} \varepsilon_{x}^{1}+A_{5}^{2} \varepsilon_{y}^{1}+A_{6}^{1} \varepsilon_{x}^{2}+A_{6}^{2} \varepsilon_{y}^{2}-B_{3}\left(T+t_{1} \dot{T}-T_{o}\right), \\
& S_{y}=A_{3}^{1} \varepsilon_{y}^{0}+A_{3}^{2} \varepsilon_{x}^{0}+A_{5}^{1} \varepsilon_{y}^{1}+A_{5}^{2} \varepsilon_{x}^{1}+A_{6}^{1} \varepsilon_{y}^{2}+A_{6}^{2} \varepsilon_{x}^{2}-B_{3}\left(T+t_{1} \dot{T}-T_{o}\right), \\
& S_{x y}=A_{3}^{3} \varepsilon_{x y}^{0}+A_{5}^{3} \varepsilon_{x y}^{1}+A_{6}^{3} \varepsilon_{x y}^{2} \\
& Q_{x z}=A_{7}^{3} \varphi_{x}, \quad Q_{y z}=A_{7}^{3} \varphi_{y} .
\end{aligned}
$$

Here, the coefficients $A_{i}^{1}, A_{i}^{2}(i=1, \ldots, 6), A_{j}^{3}(j=1, \ldots, 7)$, and $B_{k}(k=1,2,3)$ are defined by

$$
\begin{aligned}
\left\{A_{1}^{1}, A_{2}^{1}, A_{3}^{1}, A_{4}^{1}, A_{5}^{1}, A_{6}^{1}\right\} & =\int_{-h / 2}^{+h / 2} E^{1}\left\{1, z, \Psi, z^{2}, z \Psi, \Psi^{2}\right\} \mathrm{d} z \\
\left\{A_{1}^{2}, A_{2}^{2}, A_{3}^{2}, A_{4}^{2}, A_{5}^{2}, A_{6}^{2}\right\} & =\int_{-h / 2}^{+h / 2} E^{2}\left\{1, z, \Psi, z^{2}, z \Psi, \Psi^{2}\right\} \mathrm{d} z \\
\left\{A_{1}^{3}, A_{2}^{3}, A_{3}^{3}, A_{4}^{3}, A_{5}^{3}, A_{6}^{3}, A_{7}^{3}\right\} & =\int_{-h / 2}^{+h / 2} E^{3}\left\{1, z, \Psi, z^{2}, z \Psi, \Psi^{2}, \Psi^{\prime}\right\} \mathrm{d} z \\
\left\{B_{1}, B_{2}, B_{3}\right\} & =\int_{-h / 2}^{+h / 2} \beta\{1, z, \Psi\} \mathrm{d} z .
\end{aligned}
$$

By using Hamilton's principle, the governing equations can be obtained in the form

$$
\begin{gathered}
\partial_{x} N_{x}+\partial_{y} N_{x y}=0 \\
\partial_{x} N_{x y}+\partial_{y} N_{y}=0 \\
\partial_{x}^{2} M_{x}+2 \partial_{x} \partial_{y} M_{x y}+\partial_{y}^{2} M_{y}=0, \\
\partial_{x} S_{x}+\partial_{y} S_{x y}-Q_{x z}=0 \\
\partial_{x} S_{x y}+\partial_{y} S_{y}-Q_{y z}=0 .
\end{gathered}
$$


The edges of the plate are assumed to be simply supported and maintained at the reference temperature. That is,

$$
\begin{array}{ll}
w=v=\varphi_{y}=N_{x}=M_{x}=S_{x}=T=0, & \text { at } x=0, a, \\
w=u=\varphi_{x}=N_{y}=M_{y}=S_{y}=T=0, & \text { at } y=0, b,
\end{array}
$$

For the present problem, the solution for the change in temperature is sought in the form

$$
T(x, y, z, t)=e^{I \omega t} \tau(z) \sin (\lambda x) \sin (\mu y)
$$

where $\lambda=\pi / a, \mu=\pi / b$, and $I=\sqrt{-1}$. This temperature identically satisfies the boundary conditions given in (4.4) at the edges of the plate. The function $\tau(z)$ will be obtained from the solution of the heat equation (3.4). In addition, we assume the following solution form for $\left(u, v, w, \varphi_{x}, \varphi_{y}\right)$ that satisfies the boundary conditions:

$$
\left\{\begin{array}{c}
u \\
v \\
w \\
\varphi_{x} \\
\varphi_{y}
\end{array}\right\}=\left\{\begin{array}{l}
U \cos (\lambda x) \sin (\mu y) \\
V \sin (\lambda x) \cos (\mu y) \\
W \sin (\lambda x) \sin (\mu y) \\
X \cos (\lambda x) \sin (\mu y) \\
Y \sin (\lambda x) \cos (\mu y)
\end{array}\right\} e^{I \omega t}
$$

where $U, V, W, X$, and $Y$ are arbitrary parameters. Finally, we get the stress components

$$
\begin{gathered}
\sigma_{x}=-e^{I \omega t}\left\{E^{1}\left[\lambda U-z \lambda^{2} W+\Psi \lambda X\right]+E^{2}\left[\mu V-z \mu^{2} W+\Psi \mu Y\right]+\beta \tau\right\} \sin (\lambda x) \sin (\mu y)+\beta T_{o}, \\
\sigma_{y}=-e^{I \omega t}\left\{E^{2}\left[\lambda U-z \lambda^{2} W+\Psi \lambda X\right]+E^{1}\left[\mu V-z \mu^{2} W+\Psi \mu Y\right]+\beta \tau\right\} \sin (\lambda x) \sin (\mu y)+\beta T_{o}, \\
\sigma_{y z}=E^{3} e^{I \omega t} \Psi^{\prime} Y \sin (\lambda x) \cos (\mu y), \\
\sigma_{x z}=E^{3} e^{I \omega t} \Psi^{\prime} X \cos (\lambda x) \sin (\mu y), \\
\sigma_{x y}=E^{3} e^{I \omega t}[\mu U+\lambda V-2 z \lambda \mu W+\Psi(\mu X+\lambda Y)] \cos (\lambda x) \cos (\mu y) .
\end{gathered}
$$

Substitution for $T$ from (4.5) into (3.4), with aid of (4.6), gives the following partial differential equation with variable coefficients:

$$
\begin{aligned}
\frac{\mathrm{d}^{2} \tau}{\mathrm{d} z^{2}}+ & \frac{1}{\kappa} \frac{\mathrm{d} \kappa}{\mathrm{d} z} \frac{\mathrm{d} \tau}{\mathrm{d} z}-\left[\lambda^{2}+\mu^{2}+\frac{I \rho c \omega}{\kappa}\left(1-t_{2} \omega\right)\right] \tau \\
& +\frac{I \omega \beta T_{0}\left(1+I t_{3} \omega\right)}{\kappa}\left[\lambda U+\mu V-z\left(\lambda^{2}+\mu^{2}\right) W+\Psi(\lambda X+\mu Y)\right]=0 .
\end{aligned}
$$


Inserting into the above ordinary differential equation the material properties $\kappa, \rho, c$, and $\beta$ from (2.1), we obtain

$$
\begin{aligned}
& \frac{\mathrm{d}^{2} \tau}{\mathrm{d} z^{2}}+\frac{\eta_{\kappa}}{h} \frac{\mathrm{d} \tau}{\mathrm{d} z}-\left[\lambda^{2}+\mu^{2}+\frac{I \rho_{m} c_{m} \omega}{\kappa_{m}}\left(1-\omega t_{2}\right) e^{\left(\eta_{\rho c}-\eta_{\kappa}\right)((2 z+h) / 2 h)}\right] \tau \\
& \quad+\frac{I \omega \beta_{m} T_{0}\left(1+I t_{3} \omega\right)}{\kappa_{m}} e^{\left(\eta_{\beta}-\eta_{\kappa}\right)((2 z+h) / 2 h)}\left[\lambda U+\mu V-z\left(\lambda^{2}+\mu^{2}\right) W+\frac{h}{\pi} \sin \left(\frac{\pi z}{h}\right)(\lambda X+\mu Y)\right]=0,
\end{aligned}
$$

where

$$
\eta_{\kappa}=\ln \left(\frac{\kappa_{c}}{\kappa_{m}}\right), \quad \eta_{\rho c}=\ln \left(\frac{\rho_{c} c_{c}}{\rho_{m} c_{m}}\right), \quad \eta_{\beta}=\ln \left(\frac{\beta_{c}}{\beta_{m}}\right) .
$$

The exact closed form solution of (4.9) is given by

$$
\tau(z)=e^{-\eta_{\kappa} z / 2 h}\left[B_{I}(-\xi, \xi) C_{1}+B_{K}(\xi, \xi) C_{2}+\omega T_{0} \beta_{m} F(z)\right]
$$

where $C_{1}$ and $C_{2}$ are the integration constants, and the functions $B_{I}$ and $B_{K}$ are the modified Bessel's functions of the first and second kinds, respectively, in which

$$
\begin{gathered}
\xi=\frac{\sqrt{\eta_{\kappa}^{2}+h^{2}\left(\lambda^{2}+\mu^{2}\right)}}{\left|\eta_{\rho c}-\eta_{\kappa}\right|}, \\
\zeta=\frac{2 I \sqrt{\gamma \omega\left(t_{2} \omega-I\right)}}{\eta_{\kappa}-\eta_{\rho c}} e^{\left(\eta_{\rho c}-\eta_{\kappa}\right)((2 z+h) / 4 h)} .
\end{gathered}
$$

Note that the term

$$
\gamma=\frac{\rho_{m} c_{m} h^{2}}{\kappa_{m}}
$$

represents the characteristic time of heat conduction through length $h$. The function $F(z)$ is given by

$$
F(z)=B_{K}(\xi, \zeta) \int \frac{B_{I}(-\xi, \zeta) \Theta}{\Delta} \mathrm{d} z-B_{I}(-\xi, \zeta) \int \frac{B_{K}(\xi, \zeta) \Theta}{\Delta} \mathrm{d} z
$$


where

$$
\begin{aligned}
& \Delta=\kappa_{m}\left\{B_{K}(\xi, \zeta) B_{I}(1-\xi, \zeta) \sqrt{\gamma \omega\left(t_{2} \omega-I\right)}\right. \\
& \left.+B_{I}(-\xi, \zeta)\left[\sqrt{\gamma \omega\left(t_{2} \omega-I\right)} B_{K}(1+\xi, \zeta)-I \xi\left(\eta_{\rho c}-\eta_{\kappa}\right) e^{\left(\eta_{\kappa}-\eta_{\rho c}\right)((2 z+h) / 4 h)} B_{K}(\xi, \zeta)\right]\right\}, \\
& \Theta=-h e^{\left(2 z\left(2 \eta_{\beta}-\eta_{\rho c}\right)+h\left(2 \eta_{\beta}-\eta_{\kappa}-\eta_{\rho c}\right)\right) / 4 h}\left[\lambda U+\mu V-z\left(\lambda^{2}+\mu^{2}\right) W+\hbar \sin \left(\frac{\pi z}{h}\right)(\lambda X+\mu Y)\right]\left(1+I t_{3} \omega\right) \text {. }
\end{aligned}
$$

Note that the constants $C_{1}$ and $C_{2}$ are given from the temperature boundary conditions at the lower and upper surfaces of the plate

$$
\tau(z)= \begin{cases}\tau_{0}, & \text { at } z=-\frac{h}{2} \\ \tau_{1}, & \text { at } z=+\frac{h}{2} .\end{cases}
$$

\section{Numerical Results and Discussion}

We present exact results for a simply supported FG square plate subjected to a transient thermal load. Since it is common in high-temperature applications to employ a ceramic top layer as a thermal barrier to a metallic structure, we choose the constituent materials of the FG plate to be Aluminum ( $\mathrm{Al}$ ) and Silicon $(\mathrm{SiC})$ having the following material properties:

$$
\begin{aligned}
\mathrm{Al}: & E_{m}=70 \mathrm{GPa}, \quad v_{m}=0.3, \quad \alpha_{m}=23.4 \times 10^{-6} / K, \\
\kappa_{m} & =233 \mathrm{~W} / \mathrm{mk}, \quad c_{m}=896 \mathrm{~J} / \mathrm{kgK}, \quad \rho_{m}=2707 \mathrm{~kg} / \mathrm{m}^{3}, \\
\mathrm{SiC}: & E_{c}=427 \mathrm{GPa}, \quad v_{c}=0.17, \quad \alpha_{c}=4.3 \times 10^{-6} / K, \\
\mathcal{K}_{c} & =65 \mathrm{~W} / \mathrm{mK}, \quad c_{c}=670 \mathrm{~J} / \mathrm{kgK}, \quad \rho_{c}=3100 \mathrm{~kg} / \mathrm{m}^{3} .
\end{aligned}
$$

The dimensions of the simply supported FG plate are $a=b=0.25 \mathrm{~m}$. In addition, the values of different parameters are used as follows:

$$
T_{0}=1, \quad \tau_{0}=0, \quad \tau_{1}=1, \quad \omega=5 \times 10^{-4} 1 / \mathrm{s} .
$$


Table 1: Dimensionless results for FGM square plates according to various theories of thermoelasticity with different time parameters.

\begin{tabular}{lcccccc}
\hline $\mathrm{t}$ & Theory & $\mathrm{T}$ & $w$ & $\sigma_{1}$ & $\sigma_{5}$ & $\sigma_{6}$ \\
\hline \multirow{3}{*}{0.05} & C-T & 0.333122102 & 0.383966985 & 0.569639832 & 0.393610131 & -0.404103471 \\
& L-S & 0.333122105 & 0.383966988 & 0.569639832 & 0.393610133 & -0.404103471 \\
& G-L & 0.333122105 & 0.383966675 & 0.569639365 & 0.393609811 & -0.404103139 \\
\hline \multirow{3}{*}{0.10} & C-T & 0.319915220 & 0.368744318 & 0.546983655 & 0.377986612 & -0.388031140 \\
& L-S & 0.319915222 & 0.368744321 & 0.546983655 & 0.377986614 & -0.388031140 \\
& G-L & 0.319915222 & 0.368743701 & 0.546982732 & 0.377985978 & -0.388030485 \\
\hline \multirow{3}{*}{0.20} & C-T & 0.268738109 & 0.309755974 & 0.459333491 & 0.317481666 & -0.325851963 \\
& L-S & 0.268738111 & 0.309755977 & 0.459333491 & 0.317481667 & -0.325851963 \\
& G-L & 0.268738111 & 0.309754800 & 0.459331743 & 0.317480460 & -0.325850723 \\
\hline \multirow{3}{*}{0.50} & C-T & -0.018542705 & -0.021372904 & -0.032152421 & -0.022023521 & 0.022808982 \\
& L-S & -0.018542705 & -0.021372904 & -0.032152421 & -0.022023521 & 0.022808982 \\
& G-L & -0.018542705 & -0.021374846 & -0.032155303 & -0.022025512 & 0.022811027 \\
\hline \multirow{2}{*}{0.80} & C-T & -0.289521187 & -0.333711203 & -0.495370643 & -0.342166120 & 0.351416781 \\
& L-S & -0.289521189 & -0.333711205 & -0.495370643 & -0.342166122 & 0.351416781 \\
& G-L & -0.289521189 & -0.333712205 & -0.495372125 & -0.342167146 & 0.351417832 \\
\hline \multirow{2}{*}{1.00} & C-T & -0.335469789 & -0.386673003 & -0.573677254 & -0.396389968 & 0.406967624 \\
& L-S & -0.335469792 & -0.386673006 & -0.573677254 & -0.396389970 & 0.406967624 \\
& G-L & -0.335469792 & -0.386672791 & -0.573676933 & -0.396389749 & 0.406967397 \\
\hline
\end{tabular}

Numerical results are presented in terms of the nondimensional variables defined as

$$
\begin{gathered}
\mathrm{t}=\frac{t}{10^{3} \gamma}, \quad \mathrm{T}=\frac{1}{\tau_{1}} T\left(\frac{a}{2}, \frac{b}{2}, Z, t\right), \quad w=\frac{1}{h \alpha_{m} \tau_{1}} u_{3}\left(\frac{a}{2}, \frac{b}{2}, Z, t\right), \\
\sigma_{1}=\frac{1}{E_{m} \alpha_{m} \tau_{1}} \sigma_{x}\left(\frac{a}{2}, \frac{b}{2}, Z, t\right), \quad \sigma_{5}=\frac{a}{h E_{m} \alpha_{m} \tau_{1}} \sigma_{x z}\left(0, \frac{b}{2}, Z, t\right), \\
\sigma_{6}=\frac{1}{E_{m} \alpha_{m} \tau_{1}} \sigma_{x y}(0,0, Z, t), \quad Z=\frac{z}{h},
\end{gathered}
$$

The results of the classical coupled theory (C-T), Lord and Shulman's theory (L-S), and Green and Lindsay's theory (G-L) for various values of time parameter $t$ are listed in Table 1 . The relaxation times for these theories are chosen to be

$$
\begin{aligned}
& \text { C-T }: t_{1}=t_{2}=t_{3}=0, \\
& \text { L-S }: t_{1}=0, \quad t_{2}=t_{3}=0.02 \mathrm{~s}, \\
& \text { G-L }: t_{1}=0.01 \mathrm{~s}, \quad t_{2}=2 t_{1}, \quad t_{3}=0 .
\end{aligned}
$$

The dimensionless temperature $\mathrm{T}$, displacement $w$, longitudinal stress $\sigma_{1}$, transverse shear stress $\sigma_{5}$, and in-plane shear stress $\sigma_{6}$ are given at $z=0,0,1 / 2,1 / 4$, and $1 / 2$, respectively. 


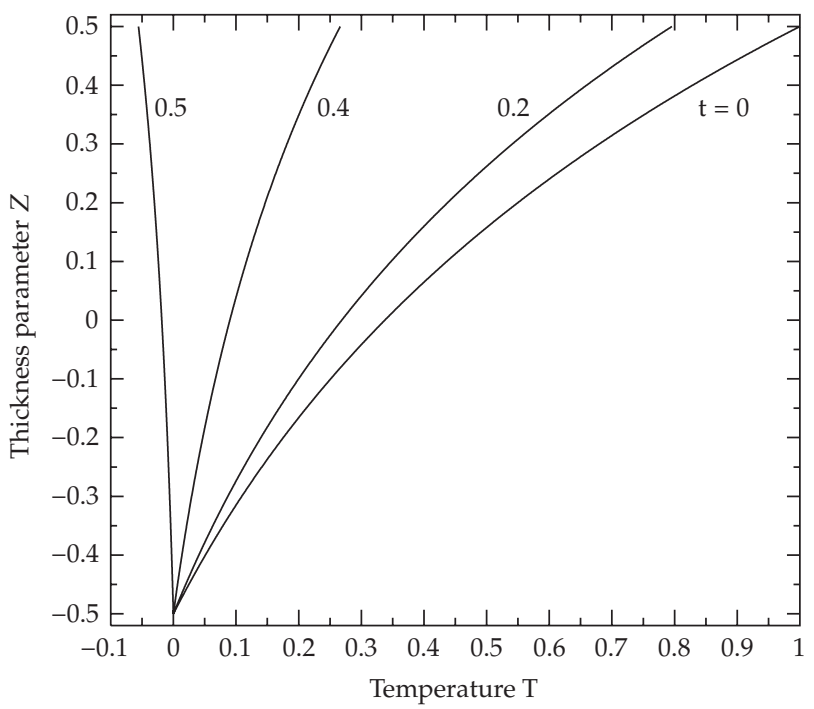

Figure 1: Through-the-thickness variation of dimensionless temperature $\mathrm{T}$ of FGM square plate for different values of the time parameter $t$.

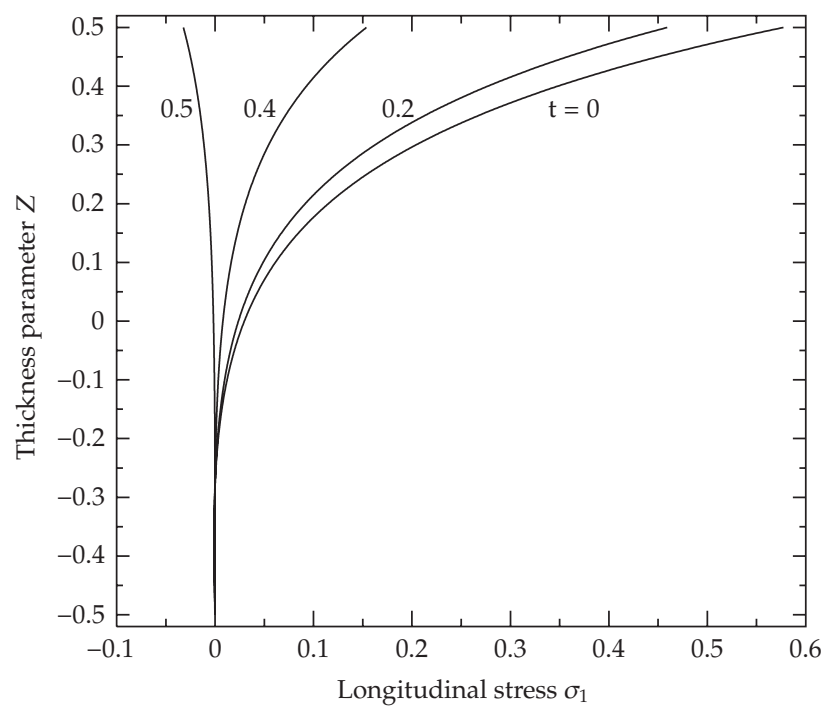

Figure 2: Through-the-thickness variation of dimensionless longitudinal stress $\sigma_{1}$ of FGM square plate for different values of the time parameter $t$.

It can be found from Table 1 that the results obtained by the L-S model agree well with those obtained by C-T whereas the G-L model gives an accurate prediction of the results that slightly differ from the above two models. The temperature given in the context of all theories may be unchanged.

It is well known that G-L theory is accurate to predict temperature, displacement, and stresses, so some results have been plotted in Figures 1-8. The through-the-thickness variation of the temperature and stresses is plotted in Figures 1, 2, 3, and 4 for different 


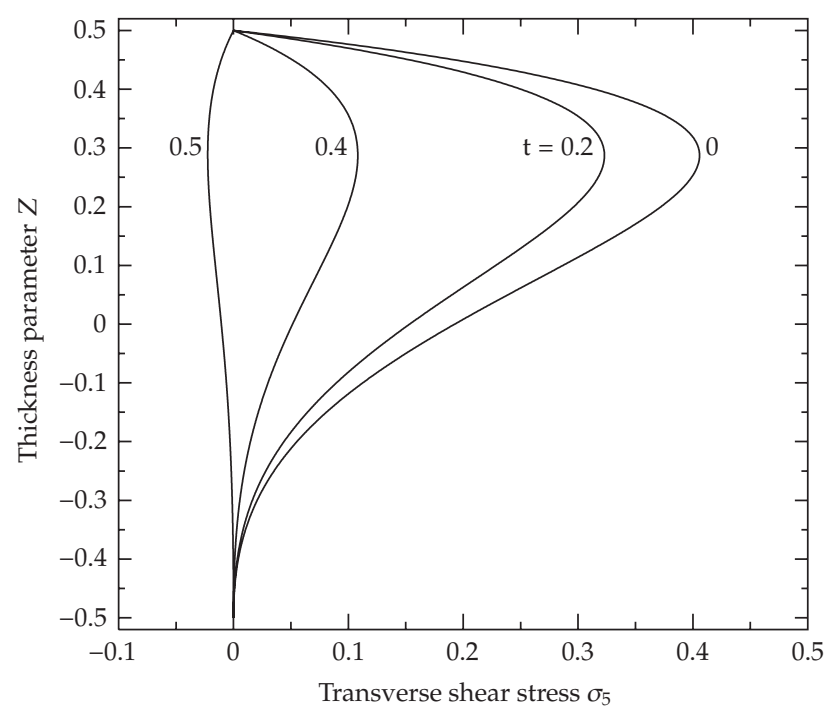

Figure 3: Through-the-thickness variation of dimensionless transverse shear stress $\sigma_{5}$ of FGM square plate for different values of the time parameter $t$.

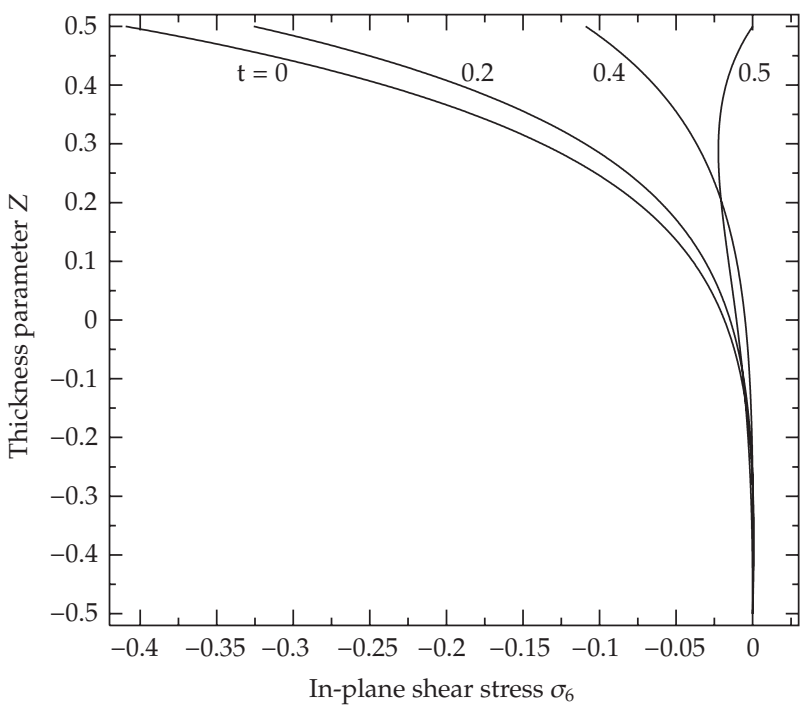

Figure 4: Through-the-thickness variation of dimensionless in-plane shear stress $\sigma_{6}$ of FGM square plate for different values of the time parameter $t$.

values of the time parameter $t$. The through-the-thickness variation of the longitudinal stress $\sigma_{1}$, transverse shear stress $\sigma_{5}$, and in-plane shear stress $\sigma_{6}$ changes significantly as a function of time. For example, at $t=0$ the magnitude of the temperature and longitudinal stress is maximum at a point on the top surface of the plate. However at the maximum values of the transverse shear stress occur at $z=0.298$ for lower values of $t$. The magnitude of the in-plane stress is maximum at a point on the top surface of the plate and it increases as $t$ increases. Since 


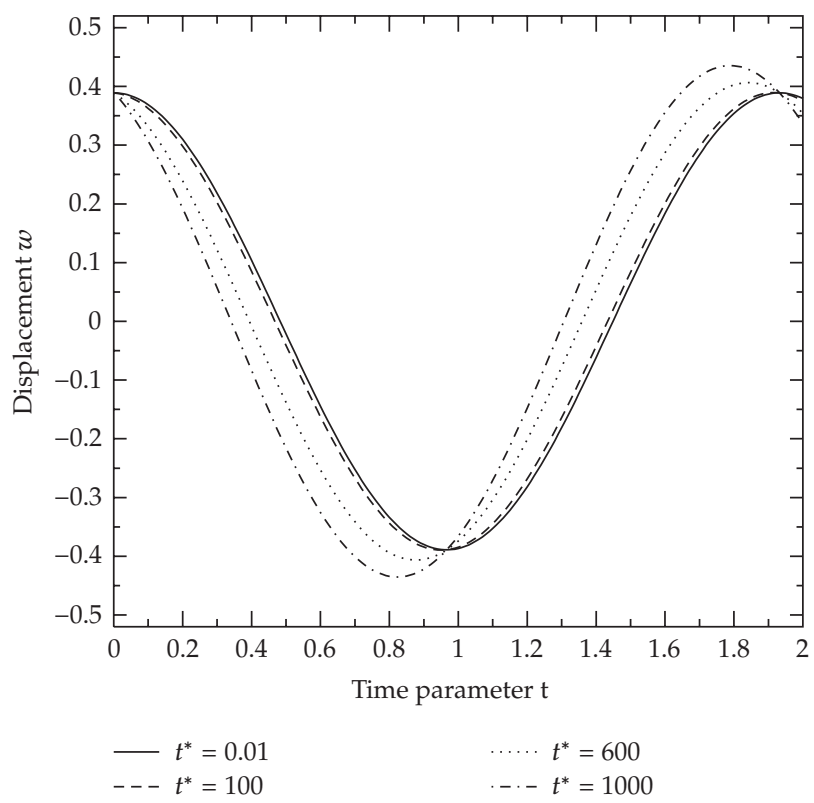

Figure 5: Dimensionless displacement $w$ versus time parameter $t$ of FGM square plate for different values of relaxation time $t^{*}$.

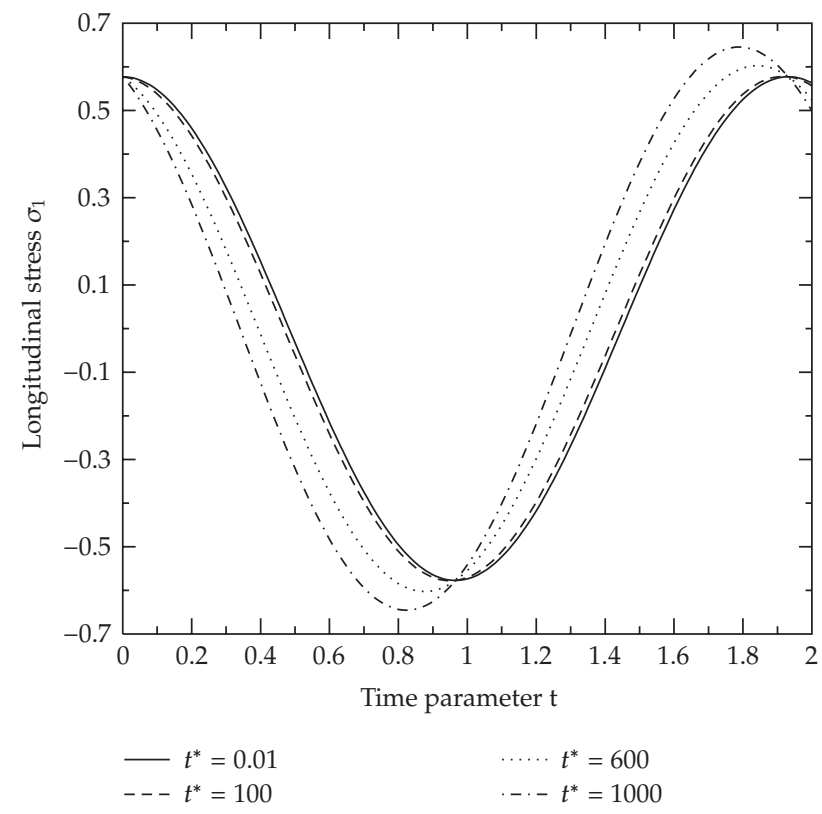

Figure 6: Dimensionless longitudinal stress $\sigma_{1}$ versus time parameter $\mathrm{t}$ of FGM square plate for different values of relaxation time $t^{*}$.

material properties and the temperature change vary through the thickness, then the throughthe-thickness variation of the longitudinal stress is nonlinear and the maximum values of the transverse shear stress dose not occur at the center of the plate. 


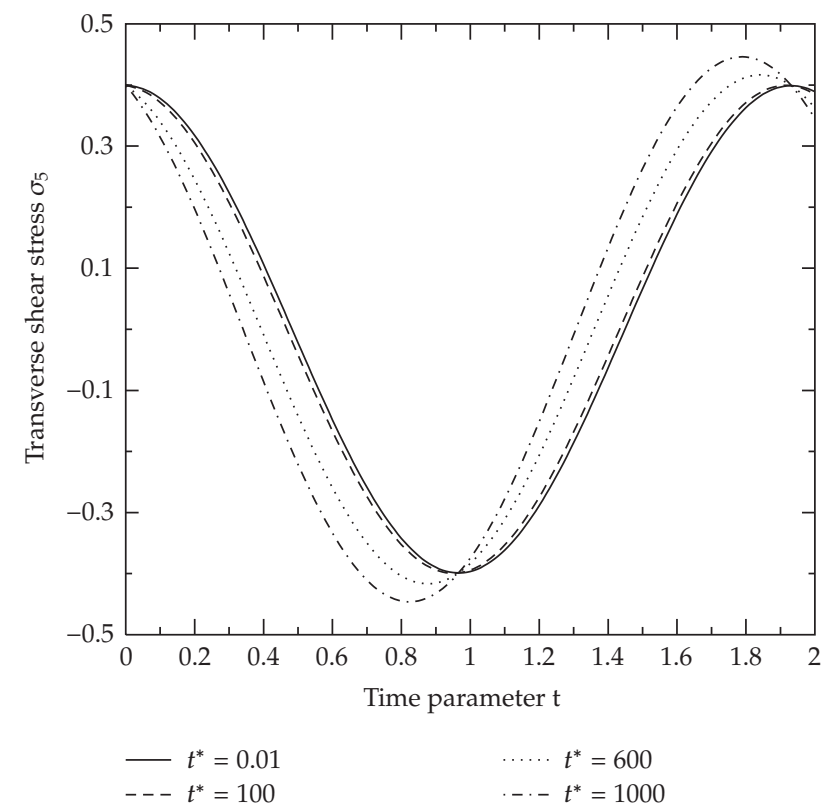

Figure 7: Dimensionless transverse shear stress $\sigma_{5}$ versus time parameter $\mathrm{t}$ of FGM square plate for different values of relaxation time $t^{*}$.

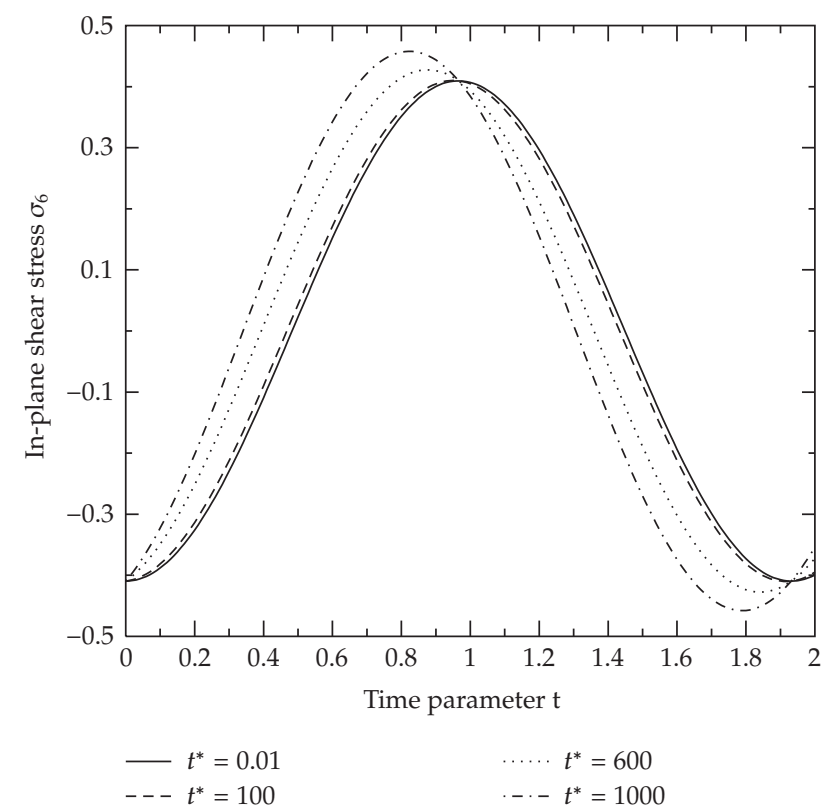

Figure 8: Dimensionless in-plane shear stress $\sigma_{6}$ versus time parameter $\mathrm{t}$ of FGM square plate for different values of relaxation time $t^{*}$.

Now, we discuss the effect of relaxation times in G-L model. Let the first relaxation time $t_{1}=t^{*}$ and the second relaxation time be double of it. Figures $5,6,7$, and 8 present the 
displacement and stresses versus the time parameter using different values of the relaxation time $t^{*}$. It is clear that all results are very sensitive to the variation of the relaxation time. The results may be inaccurate for higher values of $t^{*}$.

\section{Conclusion}

In this paper, the numerical illustrations concern quasistatic bending response of FG square plates are studied in the context of the generalized thermoelasticity theories. A refined shear deformation theory is used for this purpose. Material properties of the plate are assumed to be graded in the thickness direction according to a simple exponential law distribution in terms of the volume fractions of the constituents. An exact solution for the present problem is obtained. Numerical results are provided to show the influence of the material properties, and a temperature field on the displacement and stresses.

From these results, we can conclude that

(1) the results of G-L model give an accurate prediction comparing with those obtained by the other two models;

(2) the results of L-S model agree well with those obtained by C-T model;

(3) the temperature may be independent of the parameters used in the different theories;

(4) for higher values of the relaxation times, L-S and G-L models may be failed to get accurate solution comparing with the C-T model.

\section{Acknowledgment}

The investigators would like to express their appreciation to the Deanship of Scientific Research at King AbdulAziz University for their financial support of this study, Grant no. $180 / 428$.

\section{References}

[1] A. M. Zenkour, "A comprehensive analysis of functionally graded sandwich plates—part 1: deflection and stresses," International Journal of Solids and Structures, vol. 42, no. 18-19, pp. 5224-5242, 2005.

[2] A. M. Zenkour, "A comprehensive analysis of functionally graded sandwich plates-part 2: buckling and free vibration," International Journal of Solids and Structures, vol. 42, no. 18-19, pp. 5243-5258, 2005.

[3] A. M. Zenkour, "Generalized shear deformation theory for bending analysis of functionally graded plates," Applied Mathematical Modelling, vol. 30, no. 1, pp. 67-84, 2006.

[4] A. M. Zenkour, "Benchmark trigonometric and 3-D elasticity solutions for an exponentially graded thick rectangular plate," Archive of Applied Mechanics, vol. 77, no. 4, pp. 197-214, 2007.

[5] R. C. Wetherhold, S. Seelman, and J. Wang, "Use of functionally graded materials to eliminate or control thermal deformation," Composites Science and Technology, vol. 56, no. 9, pp. 1099-1104, 1996.

[6] S. Suresh and A. Mortensen, "Functionally graded metals and metal-ceramic composites-part 2: thermal mechanical behavior," International Materials Reviews, vol. 42, no. 3, pp. 85-116, 1997.

[7] G. V. Praveen and J. N. Reddy, "Nonlinear transient thermoelastic analysis of functionally graded ceramic-metal plates," International Journal of Solids and Structures, vol. 35, no. 33, pp. 4457-4476, 1998.

[8] H. W. Lord and Y. Shulman, "A generalized dynamical theory of thermoelasticity," Journal of the Mechanics and Physics of Solids, vol. 15, no. 5, pp. 299-309, 1967.

[9] A. E. Green and K. A. Lindsay, "Thermolasticity," Journal of Elasticity 2, vol. 2, pp. 1-7, 1972. 
[10] J. Ignaczak, “Uniqueness in generalized thermoelasticity," Journal of Thermal Stresses, vol. 2, no. 2, pp. 171-175, 1979.

[11] R. S. Dhaliwal and A. Singh, Dynamic Coupled Thermoelasticity, Hindustan Publishing, New Delhi, India, 1980.

[12] R. Dhaliwal and H. Sherief, "Generalized thermoelasticity for anisotropic media," Quarterly of Applied Mathematics, vol. 33, no. 1, pp. 1-8, 1980.

[13] D. S. Chandrasekharaiah, "Thermoelasticity with second sound," Applied Mechanics Reviews, vol. 39, pp. 355-376, 1986.

[14] R. S. Dhaliwal and H. S. Saxena, "Generalized magnetothermoelastic waves in an infinite elastic solid with a cylindrical cavity," Journal of Thermal Stresses, vol. 14, no. 4, pp. 353-369, 1991.

[15] A. E. Green and P. M. Naghdi, "Thermoelasticity without energy dissipation," Journal of Elasticity, vol. 31, no. 3, pp. 189-208, 1993.

[16] H. Sherief and R. Dhaliwal, "A uniqueness theorem and a variational principle for generalized thermoelasticity," Journal of Thermal Stresses, vol. 3, no. 2, pp. 223-231, 1980.

[17] H. Sherief and R. Dhaliwal, "Generalized thermoelasticity for anisotropic media," Quarterly of Applied Mathematics, vol. 33, no. 1, pp. 1-8, 1980/81.

[18] K. Daneshjoo and M. Ramezani, "Coupled thermoelasticity in laminated composite plates based on Green-Lindsay model," Composite Structures, vol. 55, no. 4, pp. 387-392, 2002.

[19] A. E. Green and N. Laws, "On the entropy production inequality," Archive for Rational Mechanics and Analysis, vol. 45, no. 1, pp. 47-53, 1972.

[20] R. B. Hetnarski and J. Ignaczak, "Nonclassical dynamical thermoelasticity," International Journal of Solids and Structures, vol. 37, no. 1-2, pp. 215-224, 2000.

[21] A. Bagri and M. R. Eslami, "Generalized coupled thermoelasticity of functionally graded annular disk considering the Lord-Shulman theory," Composite Structures, vol. 83, no. 2, pp. 168-179, 2008.

[22] A. S. El-Karamany and M. A. Ezzat, "Thermal shock problem in generalized thermo-viscoelasticity under four theories," International Journal of Engineering Science, vol. 42, no. 7, pp. 649-671, 2004. 


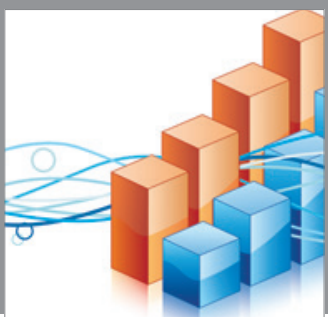

Advances in

Operations Research

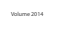

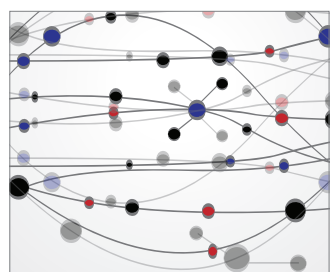

\section{The Scientific} World Journal
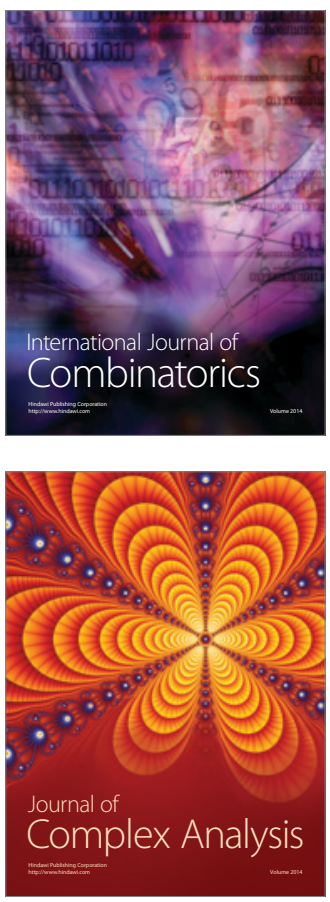

International Journal of

Mathematics and

Mathematical

Sciences
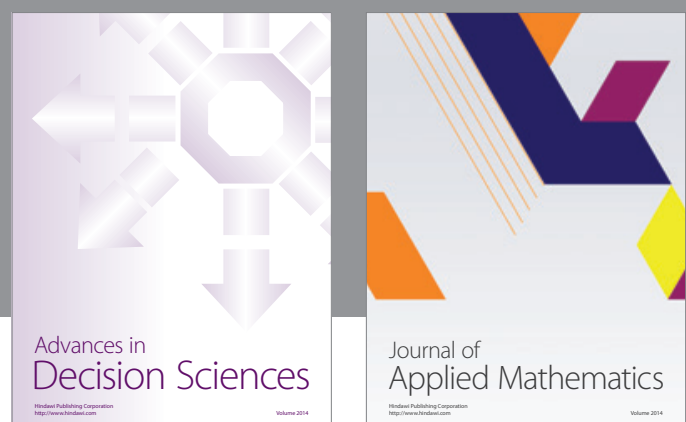

Journal of

Applied Mathematics
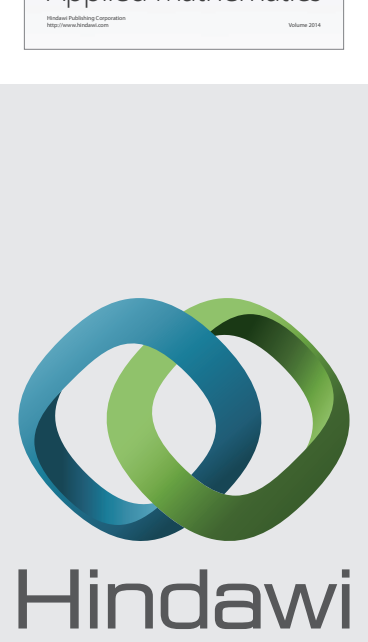

Submit your manuscripts at http://www.hindawi.com
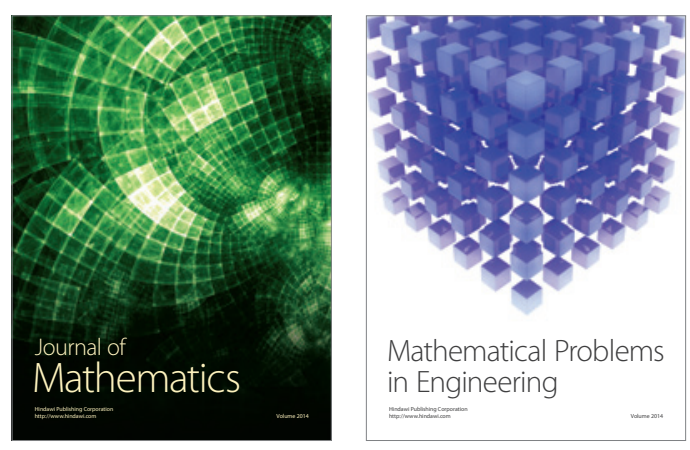

Mathematical Problems in Engineering
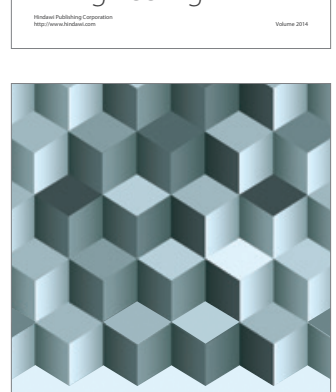

Journal of

Function Spaces
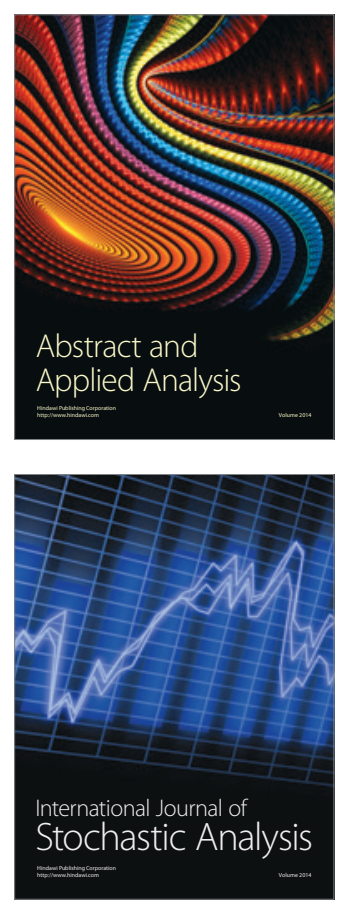

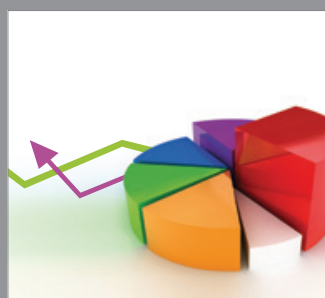

ournal of

Probability and Statistics

Promensencen
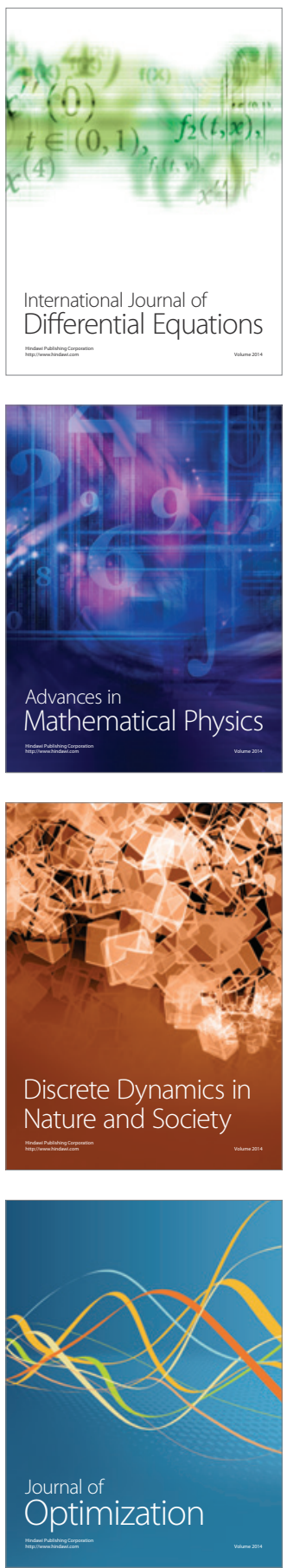